\title{
The Advent of Laser Therapies in Dermatology and Urology: Underlying Mechanisms, Recent Trends and Future Directions
}

\author{
Ho Lee \\ School of Mechanical Engineering, Kyungpook National University, Daegu 702-701, Korea \\ Yeon Uk Jeong* \\ School of Materials Science \& Engineering, Kyungpook National University, Daegu 702-701, Korea \\ Kin F. Chan \\ Fourier Biotechnologies, California 95101, USA
}

(Received June 26, 2009 : revised August 24, 2009 : accepted August 25, 2009)

\begin{abstract}
Following their applications in cardiology, ophthalmology and dentistry among others, the advent of lasers in dermatology and urology had become the success story of the past decade. Laser-assisted treatments in dermatology and urology are mainly based on the laser-induced tissue injury/coagulation and/or ablation, depending upon the desirable clinical endpoint. In this review, we discussed the underlying mechanisms of the laser induced tissue ablation. In any medical laser application, the controlled thermal injury and coagulation, and the extent of ablation, if required, are critical. The laser thermal mechanism of injury is intricately related to the selective absorption of light and its exposure duration, similarly to the laser induced ablation. The laser ablation mechanisms were categorized into four different categories (the photo-thermally induced ablation, the photo-mechanically induced ablation, the plasma induced ablation and the photoablation) and their fundamentals are herein described. The brief history of laser treatment modality in dermatology and urology are summarized.
\end{abstract}

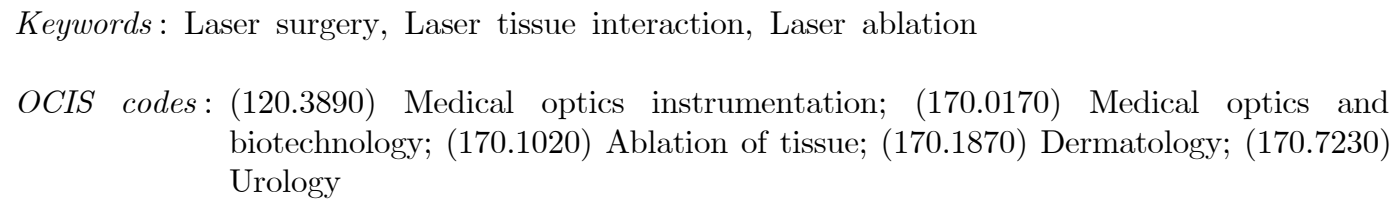

\section{INTRODUCTION}

Phototherapeutics involve the use of light to remove, injure or stimulate the body in a controlled and precise manner in order to correct or treat diseased tissues and/or to allow the body to heal itself through inflammation, proliferation and regeneration. The immediate physical interaction could be photoablative, photo-chemical, photo-mechanical or photo-thermal [1]. Both noncoherent sources (i.e. arc lamps, etc.) and coherent sources (i.e. lasers) have been explored for the phototherapeutic applications. Because of the many unique properties of the laser (such as the unidirectional, monochromatic and coherent characteristics), it has become

*Corresponding author: jeong@knu.ac.kr the preferred source of use, except under circumstances where it provides no additional value to a treatment that noncoherent sources could address or when it becomes too cost prohibitive.

In this review, we described the underlying mechanisms of photo-therapy through both photothermal and photomechanical interactions with laser energy. Recent trends and future directions of laser applications in dermatology and urology were discussed.

\section{UNDERLYING MECHANISMS OF THE LASER-ASSISTED TISSUE ABLATION}

The word "ablation" stems from the Latin ablatus 
which means "to remove". Removing portions of a tissue, using a laser with sufficient energy for a phase transfer and a chemical breakdown, is referred to as laser-induced tissue ablation. The general goal of laser ablation is the efficient removal of tissue with minimum damage to the surrounding regions [2-5]. The appropriate selection of laser parameters, which determine the ablation mechanism, is essential to achieve a successful outcome. Typically, the mechanism of laser-induced ablation falls into four categories: photo-thermally induced ablation, photo-mechanically induced ablation, plasma induced ablation, and photoablation.

\section{Photo-thermally induced tissue ablation}

The temperature rise in the laser-affected zone (where photons are directly absorbed) is one of the most common features during the interaction of laser light with matter. The photon energy of the incident laser is absorbed in the target by means of the excitation of electrons and molecules to higher energy levels. Excited particles transfer their energy to neighboring particles through a collision process, resulting in the increase of microscopic kinetic energy. Macroscopically, the increase of kinetic energy is interpreted as the temperature rise that may lead to the phase transfer of a target material. The resultant removal of the melted and vaporized material is called photothermally induced laser ablation. The ablation induced by Continuous Wave $(\mathrm{CW})$ and long pulsed (a typical pulse duration is longer than tens of microseconds) lasers falls into this category. Many medical applications of lasers (such as laser surgery in dermatology, orthopedics, dentistry, otorhinolaryngology, and urology) are based on the thermally induced ablation of soft and hard tissues [6-12].

The most challenging issue involving thermally induced ablation is the minimization of collateral damage [13-14]. The thermal energy in the laser-affected zone dissipates into surrounding regions through a heat conduction process, generating undesirable collateral damage. Since this side effect is unavoidable, researchers have tried to minimize it by selecting wavelengths and pulse durations to reduce the degree of thermal diffusion (or thermal diffusion time).

The laser lithotripsy (laser-assisted calculus fragmentation) and laser prostatectomy (laser-assisted prostate surgery) are procedures benefiting from the use of the photo-thermal mechanism. In cardiology, this mechanism has been investigated for laser angioplasty and transmyocardial laser revascularization. Recently, this mechanism is being utilized to explore cartilage reshaping and reconstruction, and most notably for dermatology.

\section{Photo-mechanically induced ablation}

The photon energy of the laser pulse can be converted to the mechanical energy (i.e. shock wave and acoustic wave) as well as the thermal energy. The laser-induced strong shock wave or the acoustic wave contributes to the material removal and the collateral mechanical damage during the ablation process. The bulk material removal induced by this mechanical energy is called photo-mechanically induced ablation. The vicinity of laser-induced craters is subjected to photomechanical collateral damages like fractures and cracks. In order to excite a stress wave that is strong enough to cause physical damage, the laser pulse duration $\left(\tau_{\mathrm{p}}\right)$ should be shorter than the acoustic diffusion time $\left(\tau_{\mathrm{a}}\right)$ which is required for the laser-induced stress wave to propagate out of the laser-affected zone.

\section{Thermoelastic Expansion}

At a relatively low energy setting, the pulse laser can induce thermoelastic expansion under the stress confinement condition illustrated in Fig. 1. For the sake of simplicity, the scattering of incident light is neglected. The fluence $\mathrm{F}\left(\mathrm{J} / \mathrm{cm}^{2}\right)$ of a laser beam inside the target, which represents the energy distribution as a function of depth (z), is determined by the incident laser fluence at the surface $\left(\mathrm{F}_{\mathrm{o}}, \mathrm{J} / \mathrm{cm}^{2}\right)$ and the absorption coefficient $\left(\mu_{\mathrm{a}}\right)$. According to Beer's law, the fluence exponentially decreases with the depth:

$$
F=F_{o} \exp \left(-\mu_{a} z\right)
$$

As mentioned in the previous section, the laser energy absorption induces a rapid heating that causes the target medium to undergo an instantaneous mechanical expansion and a compressive pressure (stress wave) is generated in the irradiated volume. The amplitude $(\mathrm{P})$ of the stress wave and the temperature rise $(\mathrm{T})$ is proportional to the product of the laser fluence $(\mathrm{F})$, the absorption coefficient $\left(\mu_{\mathrm{a}}\right)$ and the Grüneisen parameter $(\Gamma)$, swhich is a non-dimensional thermal variable [15].

$$
\begin{aligned}
& \Gamma=\frac{\beta \rho^{2}}{C_{p}} \\
& P=\Gamma \mu_{a} F=\Gamma \mu_{a} F_{o} \exp \left(-\mu_{a} z\right)=\frac{\beta \rho^{2}}{C_{p}} \mu_{a} F_{o} \exp \left(-\mu_{a} z\right)
\end{aligned}
$$

Where, $\beta$ denotes the thermal coefficient of volume expansion and $\mathrm{C}_{\mathrm{p}}(\mathrm{J} / \mathrm{gK})$ denotes the heat capacity at a constant pressure. Once the stress wave is generated, it propagates in two directions: into the target (downward) and out of the target (upward) with half amplitude of the initial value. While the downward wave (it is not depicted in Fig. 1) keeps propagating deeper without being interrupted, the upward wave is reflected at the interface between the target and surrounding medium due to the acoustic impedance mismatching. If the impedance of the medium (usually air) is much smaller than that of the target, this condition called pressure release surface condition, the reflected wave changes its 


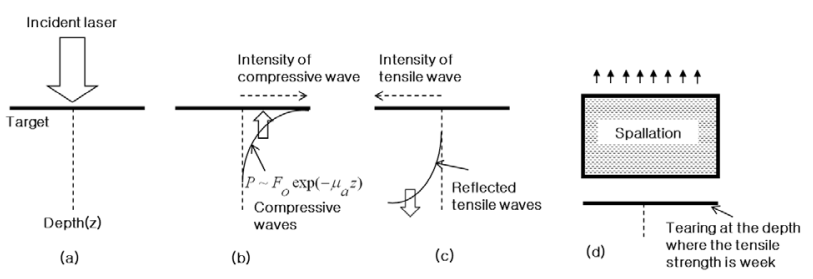

FIG. 1. Schematic illustration of the spallation process. (a) The incident light pulse, (b) The laser produces a temperature and pressure profile that follows the fluence distribution along the laser beam axis. (c) The initial wave became a tensile wave upon the reflection at the target surface and the reflected wave travels downward. (d) If the material tensile strength at a certain location is weaker than the strength of the tensile wave, spallation takes place.

sign (phase) and becomes a tensile wave (Fig. 1. (c)). If the tensile strength at a certain point in the target is weaker than the strength of the rarefactive wave, the target starts to tear apart, resulting in a bulk material removal (Fig. 1. (d)). This photomechanical ablation process is called spallation [16-18].

\section{Microexplosion}

The laser-induced microexplosion can be observed when lasers interact with the water-rich tissue. In the case of most soft tissues, the gaps between the fibers are packed by water. For hard tissues, such as bone, teeth, and calculus, the pores between the calcified materials are filled with about $10 \%$ of water. When the tissue is irradiated with an IR laser whose wavelength is close to the absorption peak of water, the vaporization of water takes place. Subsequently, the pressure inside the gaps and pores of the tissue builds up quickly, leading to the rupturing of the tissue. This process is called microexplosion [19-21].

\section{Shock wave induced ablation}

The last photomechanical ablation process is associated with shock waves that travel at supersonic speed with strong mechanical energy. Once created, the shock wave induces ablation and fractures inside the target material. Shock waves can be produced during phase explosion, bubble expansion and collapse, or plasma expansion and collapse. It has been shown that rapid laser heating of liquids, e.g., producing a temperature rise at a rate on the order of $10^{10} \mathrm{~K} / \mathrm{s}$, leads to the strong superheating of liquids [22-23]. When the liquid reaches the superheat limit, the homogeneous bubble nucleation rate increases exponentially, causing a phase explosion (also termed "explosive boiling") that induces strong shock waves. High power laser-induced plasma (a gaseous state of high temperature ions) also produces the shock wave while it expands and collapses [24-25]. The mechanism related to the plasmas is dis-

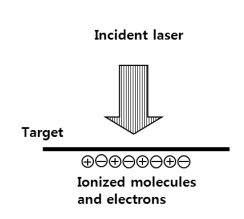

(a)

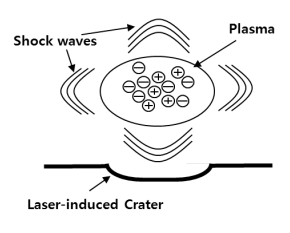

(b)

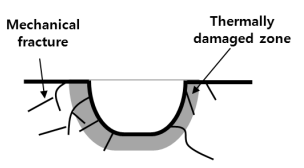

(c)
FIG. 2. The schematic illustration of plasma-induced ablation. (a) The initiation of optical breakdown (ionization process) (b) The plasma expansion and the formation of shock waves (c) Additional ablation and collateral damage around the crater.

cussed in the next section. Short pulsed laser lithotripsy using the Dye laser and Nd:YAG (whose pulse duration is about $1 \mu \mathrm{sec}$ ) is known to break the human calculus with laser-induced shock waves [26-27].

\section{Plasma-induced ablation}

The plasma is the fourth physical phase, described as a cloud of high energy gas and ions. The initiation and consequences of laser-induced plasma are illustrated in Fig. 2. When the target is exposed to the extremely high irradiance (typical pulse duration is less than $1 \mu$ sec and fluence rate is more than $10^{10} \mathrm{~W} / \mathrm{cm}^{2}$ ), a phenomenon called optical breakdown occurs [5, 28-30]. The laser creates numerous ionized molecules and free electrons. These ionized molecules and electrons are ejected from the surface and form a localized cloud called plasma. Once the plasma is generated, its complete absorption of the laser beam acts as a shield, preventing laser energy deposition to the target surface. As a result of this, the ablation yield saturates at high pulse energy, limiting the maximum ablation rate during the laser-induced material process. As mentioned in the previous section, the rapid expansion of plasma can induce shock waves that cause additional photomechanical ablation and damage. Due to the high temperature of the plasma, collateral thermal damage may also occur during plasma expansion.

The ionization can be triggered by three distinct mechanisms. In the instance of a nanosecond pulse, the thermionic process initiates the plasma. For Ultra Short Pulse Laser (USPL) process, either (or both) the multiphoton or tunneling ionizations are considered the triggering mechanism. Once the seed electrons and ions are generated through either mechanism, the number of free electrons can be multiplied by the subsequent inverse Bremsstrahlung and avalanche ionization process.

\section{Thermionic emission}

The emission of electrons or ions by highly heated substances is called the thermionic emission and the charged particles are called thermions. When a nanosecond pulse interacts with a target, the photon energy 
is converted to the kinetic energy of electrons (macroscopically, the heat energy) and subsequently, a number of thermions are emitted. During the interaction between the Q-switched nanosecond pulse and targets, such as tissue or industrial material, the plasma is initiated by means of a thermionic process [29-31]. Usually, thermal and mechanical damage accompany this process.

\section{Ultrashort pulse laser ablation}

An ultrashort pulse laser (USPL) emits pulses with a duration less than or comparable to the time required for an energy exchange between electrons and material lattices. As a result, the interaction between USPL and material can be highly localized, allowing more precise ablation to be achieved. Typical heat diffusion time in metal is a few picoseconds; in semiconductors and dielectrics, it is in the range of tens or hundreds of picoseconds. It is hypothesized that USPL ablation is initiated by multiphoton and tunneling ionization, followed by an electron avalanche. Consequently, the ablation threshold and rate are less sensitive to linear absorption of material and are governed by a nonlinear interaction between the laser pulse and the material.

The multiphoton ionization in a dielectric substance whose bandgap energy ( $\Delta \mathrm{E}$ : the energy difference between the conduction and covalence band) is higher than the single photon energy of incident light is depicted in Fig. 3. The bandgap energy of the substance in the figure is assumed to be $3 \mathrm{eV}$ and the photon energy of incident light is set to $1 \mathrm{eV}$ (corresponding to the wavelength of $1.24 \mu \mathrm{m}$ ). Originally, most electrons are bound in the covalence band (stable or lower energy state). When an electron absorbs three photons simultaneously, the electron crosses the bandgap, becoming a free electron called a seed electron. Since the ionization requires the simultaneous absorption of multiple photons, this process is called multiphoton ionization [32-35]. In order for multiphoton ionization to take place, an extremely large number of photons (provided by a high power short pulse duration laser) are needed; the greater the

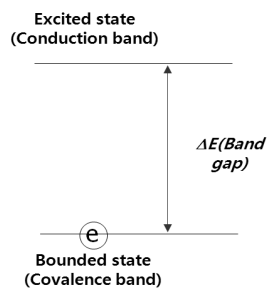

(a)

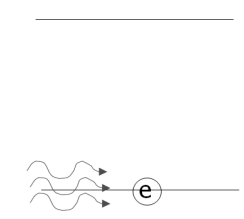

(b)

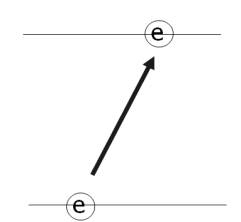

(c)
FIG. 3. The schematic illustration of the multiphoton ionization. (a) An un-excited electron resides in the bounded state. (b) The electron absorbs the three photons simultaneously. (c) As the result of the multi-photon absorption, the electron jumps to the excited state and become the free electron.

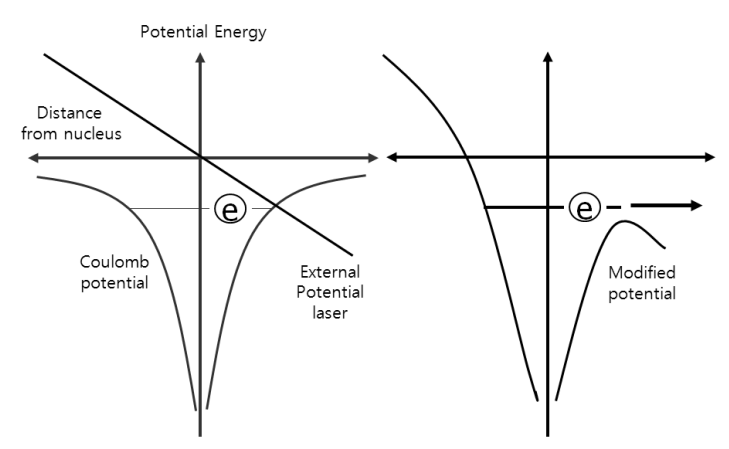

FIG. 4. The schematic illustration of the tunneling ionization.

number of photons, the higher the probability of simultaneous photon absorption.

The plasma can be initiated by another process called tunneling ionization (or field ionization) that is illustrated in Fig. 4 [36-37]. The energy states of electrons are described differently from the multiphoton ionization process for the purpose of clarity. In the absence of external fields, the energy of a bounded electron is described by the Coulomb potential. The energy of an electron at a certain distance is indicated by the horizontal line. When a strong electric field, due to the laser irradiation (shown as the straight line) is applied, the original potential energy can be modified. The bounded electron is now able to escape the potential well and become a free electron (seed electron).

Once seed electrons (excited through either multiphoton or tunneling ionization) are generated, they absorb additional photons through a process called inverse Bremsstrahlung. These seed electrons collide with nearby unexcited electrons, transferring part of their energy to neighboring electrons (this process is called collision ionization) [38-39]. Through this collision process, the number of free electrons is multiplied, eventually forming the plasma.

The potential application of USPL has been demonstrated in many fields, including pulsed laser deposition of thin films, micro-machining, and biomedical applications [40-42]. Among many practical usages, USPL application in the biomedical area is promising. A recent study has shown that an USPL system that can make clean and precise cuts in the human cornea [41]. Feasibility studies for dentistry, bone drilling, and neurosurgery have also been carried out [43-45].

\section{Photoablation}

A single photon energy of UV light ( $3 \mathrm{eV}$ at $\lambda=400$ $\mathrm{nm}$ ) is higher than the dissociation energy of common chemical bondings. Upon the UV light irradiation, the chemical bonding of the target can be broken by direct photon energy absorption, leading to material removal. This kind of material removal is called photoablation. 
Photoablation can achieve a precise material removal with minimal thermal damage to neighboring regions. Refractive corneal surgery and most semiconductor machining processes (such as lithography) are based on UV laser-induced photoablation (ArF Excimer laser operating at $193 \mathrm{~nm})$ [46-47].

\section{TRENDS AND FUTURE DIRECTION OF LASERS IN DERMATOLOGY}

Laser applications in dermatology have been a phenomenon during the past decade. Photothermal interaction below the vaporization threshold results in nonablative injury, while above this threshold results in ablative resurfacing.

Through selective photothermolysis [48], specific wavelengths which are preferentially absorbed by certain chromophores, have been selected to treat port wine stain, haemangioma, and other vascular diseases (chromophore: hemoglobin), and hair removal (chromophore: melanin).

In addition to the wavelength selection based upon the target chromophore, longer wavelengths become more preferential if the target is deeper inside the skin, as high scattering coefficients at shorter wavelength limits their penetration through the bulk tissue. Another important parameter to consider is the laser pulse duration. Both the dimension and absorption coefficient of the target help define the pulse duration to be used, as the proper thermal relaxation time [2] is necessary to damage the target with minimal collateral damage to the adjacent tissue. Typical pulse durations for most targets in dermatology range from a few milliseconds to hundreds of milliseconds. On occasion, Q-switched pulses in the nanosecond ranges are used to target pigments during tattoo removal. In conjunction with the pulse duration, the proper dosage or fluence in $\mathrm{J} / \mathrm{cm}^{2}$ was required to produce the optimal temperaturetime relation based on the Arrhenius model to damage the target [2]. Traditionally, 'damaged target' meant coagulation, such as the coagulation of hemoglobin/ blood vessel or pigmented lesions. However, the recent development has improved the understanding of subcoagulative tissue damage and its clinical benefits. For example, the temperature-time relation resulting in the induction of heat shock protein could produce or enhance the wound healing process around the ablated and/or coagulated zone [54]. In the laser bulk heating and dermal coagulation of the dermis, superficial cryogenic or contact cooling is used to protect the epidermis; this treatment modality purportedly stimulates collagen remodeling [49].

Full resurfacing (chromophore: water) is achieved with bulk superficial ablation of the epidermis and dermis with a minimal layer of coagulation zone to maintain haemostasis. This is often done with the $\mathrm{CO}_{2}$ and pulsestacked Er:YAG lasers. In laser bulk heating and dermal coagulation of the dermis, superficial cryogenic or contact cooling is used to protect the epidermis; this treatment modality purportedly stimulates collagen remodeling [49]. The former removes superficial pigmentary lesions and 'flattens' fine lines and rhytids, while the latter reduces rhytids and furrows by an increased volume of the underlying dermal collagen.

Unfortunately, full resurfacing produces unnatural skin tone and texture while also resulting in significant downtime and an increased risk profile for clinical side effects. Dermal bulk heating does not address solar lentigos or other pigmentary lesions, while having both questionable clinical efficacy and high risks of adverse side effects when not performed properly.

As a result, fractional photothermolysis [50] and fractional resurfacing [51] became the best solutions for resurfacing and dermal remodeling while offering a comfortable treatment modality with a low side-effects profile. Fractional photothermolysis and resurfacing produce microscopic thermal zones (In the ablative mode, termed microscopic treatment zones) - or MTZs that can be delivered very rapidly depending on the enabling technologies. Each MTZ consists of a vertical column of deep coagulative or ablative zones beyond $1.5 \mathrm{~mm}$ from the stratum corneum. The first commercially available fractional photothermolysis system is Fraxelff from Solta Medical. The Fraxel can deliver MTZs as fast as $3000 \mathrm{MTZ} / \mathrm{sec}$, and completing a full face treatment with minimal discomfort within 15-20 min. The Fraxel handpiece also comes with a feedback sensory system - the Intelligent Optical Tracking System (IOTS) that controls real-time dosage delivery as a function of physicians' handspeed - a unique technology in the laser dermatology industry. The ablative modality, termed Ablative Fractional Resurfacing (AFR) is developed with a high power $\mathrm{CO}_{2}$ laser [52], while the nonablative modality - Nonablative Fractional Resurfacing (NFR) is enabled with a $1550 \mathrm{~nm}$ (Fraxel re:store) or $1410 \mathrm{~nm}$ (Fraxel re:fine) laser [51]. Other fractional solutions that can be found in the market include the Lutronic MOSAIC \& $\mathrm{eCO}_{2}$, Lasering Mixto, Alma Pixel, Palomar Lux1540 \& LuxIR, etc.

AFR is uniquely able to remove diseased or sundamaged skin, such as solar elastosis, scar tissue, etc. Both the AFR and NFR are capable of normalizing pigmentary lesions, improving skin tone and texture, and promoting long-term dermal remodeling. The superficial improvement is achieved through the rapid epidermal cell turnover. Post AFR or NFR treatment, microscopic epidermal necrotic debris (MEND) is formed on each MTZ. The subsequent exfoliation or desquamation removes abnormally pigmented areas, replacing the necrotic and adjacent zone with normal and healthy epidermal cells, a process termed biological resurfacing [53]. Long-term 
dermal remodeling lasts several months post treatment with pro-collagen chaperone (hsp47) expression and direct evidence of neo-collagenesis [54].

Within the past two years, many non-invasive devices for circumferential, fat and cellulite reduction have penetrated the aesthetic market. The current state of the art for treating these indications remains questionable at best. The application and usage of these devices can be dramatically increased with well-designed clinical studies that demonstrate the definitive clinical efficacy with statistically significant enhancement in their results compared to a placebo. Hence, the race to introducing a novel treatment modality that truly addresses indications related to subcutaneous imperfection remains wide open.

\section{TRENDS AND FUTURE DIRECTION OF LASERS IN UROLOGY}

Since the first trial of a ruby laser on the calculus fragmentation in $60 \mathrm{~s}$, many studies had been pursued for the laser-assisted soft and hard tissue surgery in urology. It is generally accepted that the laser lithotripsy and laser prostatectomy are two most successful laser treatment modalities in urology.

Various types of electromagnetic waves including the microwave, radio frequency, and laser have been used to treat the abnormally enlarged prostate (Benign Prostatic Hyperplasia (BPH)). Among above mentioned modalities, the laser ablation-based treatment is called the laser prostatectomy [55-57]. The laser prostatectomy is performed by shooting the pulse laser to the abnormal prostate and enlarging the passage for the urine. The $\operatorname{Ho:YAG}(\lambda=2.12 \mu \mathrm{m})$ laser and $\operatorname{KTP}$ laser $(\lambda=532 \mathrm{~nm})$ have been used endoscopically for the prostatectomy and the laser prostatectomy is known for its superior haemostatic characteristics compared to the conventional transurethral resection.

The first generation of the clinical laser lithotripsy is based on the laser induced shock wave. The flashlamp pumped dye laser $(\lambda=504 \mathrm{~nm}$ and pulse duration $\left.\left(\tau_{p}\right)=1 \mu \mathrm{s}\right)$ and the frequency double double pulse Nd:YAG laser $\left(\lambda_{1}=532 \mathrm{~nm}, \lambda_{2}=1064 \mathrm{~nm}, \tau_{p}=1.2 \mu \mathrm{s}\right.$ at $1064 \mathrm{~nm}$ ) has been employed as the laser source and the laser beam was delivered endoscopically through the optical quartz fibers $[12,58]$. Under the stress confined conditions, the one microsecond pulse generates the strong shock wave. The induced shock wave fragments the calculus by means of the photo-mechanical effect and this treatment modality was referred as laser-induced shock wave lithotriptor. The laser-induced shock wave lithotriptor was used for a certain group of calculus and it produced relatively large fragments. Because of these limitation, the laser-induced shock wave lithotriptor was replaced by the Ho:YAG laser lithotriptor.
The second generation of laser lithotriptor was built based on the Ho:YAG laser operating at the free running mode[59]. The wavelength of the Ho:YAG laser is $2.12 \mu \mathrm{m}$ and the pulse duration is a few hundred microseconds. The endoscopically delivered Ho:YAG pulse is strongly absorbed by the calculus and it mainly fragments the calculus through the photo-thermal process. Because of its high absorption in water and the mineral components of calculus, the Ho:YAG laser lithotriptor can fragment most kinds of calculus. It is the most popularly used laser lithotriptor in the clinic these days.

Two different laser sources have been considered as the third generation lithotriptor. The fiber based thulium laser operates at $1.94 \mu \mathrm{m}$ with the pulse duration of 20 msec. It was expected that the thulium laser based lithotriptor can be more compact compared to the Ho:YAG laser lithotriptor because the thulium laser is based on the fiber system. The Fried group demonstrated that the thulium laser can fragment different types of calculus, however the efficacy of the thulium lithotriptor is currently limited by the lower maximum power and lower absorption coefficient compared to the Ho:YAG laser [60].

A few feasibility studies of Er:YAG laser has revealed that Er:YAG laser can fragment the calculus more efficiently than Ho:YAG due to its superior absorption by the calculus [62]. The Fig. 5 demonstrates the superior drilling effect of Er:YAG laser over the Ho:YAG laser. When the similar pulse energy was used, the Er:YAG laser drilled much deeper hole than the Ho:YAG laser.

The free running Er:YAG operates at the wavelength of $2.94 \mu \mathrm{m}$ and the pulse duration is about 300

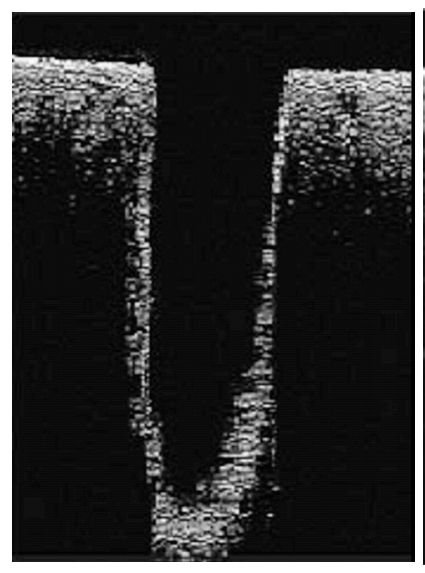

(a)

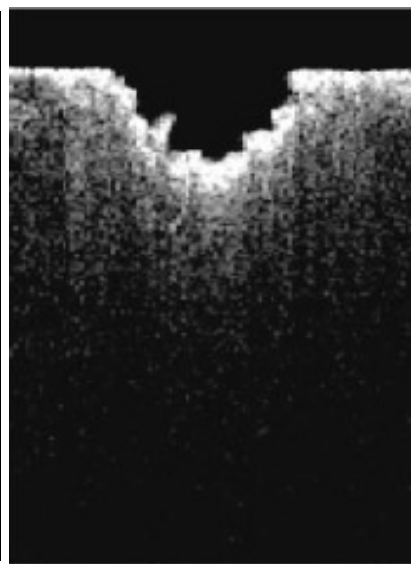

(b)
FIG. 5. Vertical cross-sectional topography (taken with the optical coherence topography) of Er:YAG and Ho:YAG laser-induced craters on a calculus. (a) Er:YAG laserinduced crater. Five pulses were irradiated with the fluence of $668 \mathrm{~J} / \mathrm{cm}^{2}$ per pulse. (b) Ho:YAG laser-induced crater. Five pulses were irradiated with the fluence of 578 $\mathrm{J} / \mathrm{cm}^{2}$ per pulse. The pictures are reprinted from [61]. 
usec. The Er:YAG laser-induced fragmentation of calculus is governed by the photo-thermal process as the Ho: YAG lithotriptor. In spite of its advantage over the Ho:YAG laser, the implementation of Er:YAG laser lithotriptor is hindered by the optical delivery system. The germanium and sapphire based fibers had been tested for the endoscopic delivery of Er:YAG laser, however these fiber did not meets the requirement of the endoscopic lithotriptor [62-66]. Because these fibers were too brittle or had low melting temperature, the tip of fibers were not able to sustain the strong thermal/mechanical interaction of the laser and calculus. In order to overcome the limitations of bare fibers, the hybrid fibers based on germanium and the hollow waveguide are currently being tested. The hybrid fiber delivery system installed the protective cap at the tip of the germanium or the hollow waveguide so that the fiber tip can be protected during the laser-calculus interaction.

The proper endoscopy delivery fiber delivery system is expected to open a new market for the Er:YAG laser in the field of laser lithotripsy. In addition to the hard tissue surgery, the Er:YAG laser can be also employed in the soft tissue surgery due to its precise incision capacity. When the Er:YAG laser is combined with the Ho:YAG laser, the Er:YAG laser can be used for the incision and the Ho:YAG can be utilized for the surgery that required the volumetric coagulation of soft tissue such as the benign prostatic hyperplasia.

\section{CONCLUSIONS}

The laser-assisted surgery is mainly based on the laser-induced tissue ablation. We have discussed four different mechanisms that can initiate the tissue removal from the surface. We also introduced some laser therapies related to the each mechanism. However, it should be noted that most laser-induced ablations are the result of a combination of two or more mechanisms described above. For example, the ejection of melted and vaporized material (photothermally-induced products) is driven by a high pressure field (photomechanicalinduced product) which is built in the crater. Due to its high energy, laser-induced plasma always causes additional thermal and mechanical effects in the vicinity of a crater. In addition, most photomechanical mechanisms start with the heating of the target, which is a photothermal effect. Because of this complicated nature of the laser-induced tissue removal, it is essential to understand the comprehensive role of various laser parameters (the wavelength, the pulse duration, the pulse energy and so on) in order to achieve the successful treatment outcome.

Speaking of the future of the laser therapy, the aesthetic dermatology and less-invasive urology surgery are particularly attractive as a business model, as the regulatory obstacle is not formidable, often allowing rapid product-to-market cycles, and does not involve insurance reimbursement (in the U.S.).

The challenges and hurdles in other medical fields in terms of clinical safety and efficacy, insurance reimbursement, and regulatory issues are more bountiful. Fortunately the present climate is likely to drive consumer pragmatism. Below the turbulent surface of economic turmoil, it appears that the next revolution in phototherapeutics is in the works. We are likely to experience the reemergence of new laser- or light-assisted therapeutic modalities in other medical fields that will garner tremendous attention in the near future.

\section{ACKNOWLEDGMENT}

This work was supported by the Grant of the Korean Ministry of Education, Science and Technology (The Regional Core Research Program/Medical Convergence Technology Development Consortium for Anti-aging and Well-being)

\section{REFERENCES}

1. S. L. Jacques, "Laser-tissue interactions: photochemical, photothermal, and photo mechanical," Surg. Clin. North Am. 72, 531-558 (1992).

2. A. J. Welch and M. J. C. van Gemert, Optical-thermal Response of Laser-irradiated Tissue (Plenum Press, New York, USA, 1995), Chapter 21-25.

3. M. H. Niemz, Laser-tissue Interactions: Fundamental and Applications (Springer, Berlin, Germany, 1996).

4. K. F. Chan, "Pulsed infrared laser ablation and clinical applications," Ph.D. Dissertation, Univ. Texas Austin (2000).

5. H. Lee, "Pulsed laser-induced material ablation and its clinical applications," Ph.D. Dissertation, Univ. Texas Austin (2000).

6. J. S. Nelson, "In this issue. Dermatologic laser surgery," Lasers Surg. Med. 26, 105-107 (2000).

7. M. B. T. Alora and R. R. Anderson, "Recent developments in cutaneous lasers," Lasers Surg. Med. 26, 108-118 (2000).

8. M. Sato, M. Ishihara, T. Arai, T. Asazuma, T. Kikuchi, T. Hayashi, T. Yamada, M. Kikuchi, and K. Fujikawa, "Use of a new ICG-dye-enhanced diode laser for percutaneous laser disc decompression," Lasers Surg. Med. 29, 282-287 (2001).

9. J. G. Sulewski, "Historical survey of laser dentistry," Dent. Clin. North Am. 44, 717-752 (2000).

10. B. M. Lippert, S. Gottschlich, C. Kulkens, B. J. Fol, H. Rudert, and J. A. Werner, "Experimental and clinical results of Er:YAG laser stapedotomy," Lasers Surg. Med. 28, 11-17 (2001).

11. P. Janda, R. Sroka, R. Baumgartner, G. Grevers, and A. Leunig, "Laser treatment of hyperplastic inferior nasal turbinates: a review," Lasers Surg. Med. 28, 404-413 (2001). 
12. K. F. Chan, T. J. Pfefer, J. M. H. Teichman, and A. J. Welch, "A perspective on laser lithotripsy: the fragmentation processes,” J. Endourol. 15, 257-273 (2001).

13. E. V. Ross, Y. Domankevitz, M. Skrobal, and R. R. Anderson, "Effects of $\mathrm{CO}_{2}$ laser pulse duration in ablation and residual thermal damage: implications for skin resurfacing," Lasers Surg. Med. 19, 123-129 (1996).

14. T. J. Pfefer, B. Choi, G. Vargas, K. M. McNally, and A. J. Welch, "Pulsed laser-induced thermal damage in whole blood,” T. ASME. J. Biomech. Eng. 122, 196-202 (2000).

15. A. A. Oraevsky, S. L. Jacques, and F. K. Tittel, "Measurement of tissue optical properties by time-resolved detection of laser-induced transient stress," Appl. Opt. 36, 402-415 (1997).

16. I. Itzkan, D. Albagli, M. L. Dark, L. T. Perelman, C. von Rosenberg, and M. S. Feld, "The thermoelastic basis of short pulsed laser ablation of biological tissue," Proc. Natl. Acad. Sci. USA 92, 1960-1964 (1995).

17. J. T. Walsh and T. F. Deutsch, "Pulsed $\mathrm{CO}_{2}$ laser ablation of tissue: effect of mechanical properties," IEEE Trans. Biomed. Eng. 36, 1195-1201 (1989).

18. A. A. Oraevsky, S. L. Jacques, and F. K. Tittel, "Mechanism of laser ablation for aqueous media irritated under confined-stress conditions,” J. Appl. Phys. 78, 1281-1290 (1995).

19. J. T. Walsh and T. F. Deutsch, "Pulsed $\mathrm{CO}_{2}$ laser tissue ablation: measurement of the ablation rate," Lasers Surg. Med. 8, 264-275 (1988).

20. B. Majaron, P. Plestenjak, and M. Lukac, "Thermomechanical laser ablation of soft biological tissue: modeling the micro-explosions," Appl. Phys. B 69, 71-80 (1999).

21. J. P. Cummings and J. T. Walsh, "Tissue tearing caused by pulsed laser-induced ablation pressure," Appl. Opt. 32, 494-503 (1993).

22. R. Kelly and A. Miotello, "Comments on explosive mechanisms of laser sputtering,” Appl. Surf. Sci. 96-98, 205-215 (1996).

23. E. E. B. Campbell, D. Ashkenasi, and A. Rosenfeld, "Ultra-short-pulse laser irradiation and ablation of dielectrics,” Mater. Sci. Forum. 301, 123-144 (1999).

24. V. E. Gusev and A. A. Karabutov, Laser Optoacoustics (AIP Press, New York, USA, 1993).

25. A. Vogel, P. Schweiger, A. Frieser, M. N. Asiyo, and R. Birngruber, "Intraocular Nd:YAG laser surgery: lighttissue interaction, damage range, and reduction of collateral effects,” IEEE J. Quantum Electron. 26, 2240-2260 (1990).

26. S. P. Dretler, "Laser lithotripsy: a review of 20 years of research and clinical applications,” Lasers Surg. Med. 8, 341-356 (1988).

27. K. Rink, G. Delacretaz, and R. P. Salathe, "Fragmentation process of current laser lithotriptors," Lasers Surg. Med. 16, 134-146 (1995).

28. D. Baurele, Laser Processing and Chemistry (Springer, Berlin, Germany, 1996), Chapter 6.

29. P. Nelson, P. Veyrie, M. Berry, and Y. Durand, "Experimental and theoretical studies of air breakdown by intense pulse of light," Phys. Lett. 13, 226-228 (1964).

30. D. W. Fradin, N. Bloembergen, and J. P. Letellier, "Dependence of laser-induced breakdown field strength on pulse duration,” Appl. Phys. Lett. 22, 635-637 (1973).

31. J. P. Ready, Effects of High-power Laser Radiation (Academic, New York, USA, 1971), pp. 133-143.

32. J. P. Ready, Effects of High-power Laser Radiation (Academic, New York, USA, 1971), pp. 215-217.

33. A. Vogel, J. Noack, G. Huettmann, and G. Paltauf, "Femtosecond-laser-produced low-density plasmas in transparent biological media: a tool for the creation of chemical, thermal, and thermomechanical effects below the optical breakdown threshold," Proc. Soc. Photo. Opt. Instrum. Eng. 4633A, 23-37 (2002).

34. A. Vogel, K. Nahen, and D. Theisen, "Plasma formation in water by picosecond and nanosecond Nd:YAG laserpulses. I. Optical breakdown at threshold and superthreshold irradiance,” IEEE J. Quantum Electron. 2, 847-860 (1996).

35. P. P. Pronko, P. A. VanRompay, C. Horvath, T. Juhasz, X. Liu, and G. Mourou, "Avalanche ionization and dielectric breakdown in silicon with ultrafast laser pulses,” Phys. Rev. B 58, 2387-2390 (1998).

36. M. Lenzner, J. Krüger, S. Sartania, Z. Cheng, C. Spielmann, G. Mourou, W. Kautek, and F. Krausz, "Femtosecond optical breakdown in dielectrics," Phys. Rev. Lett. 80, 4076-4079 (1998).

37. A. C. Tien, S. Bakus, H. Kapteyn, M. Murnane, and G. Mourou, "Short-pulse laser damage in transparent materials as a function of pulse duration," Phys. Rev. Lett. 82, 3883-3886 (1999).

38. M. Bass and H. H. Barrett, "Avalanche breakdown and the probabilistic nature of laser-induced damage," IEEE J. Quantum Electron. QE-18, 338-343 (1983).

39. D. W. Fradin, E. Yablonovitch, and M. Bass, "Confirmation of an electron avalanche causing laser-induced bulk damage at $1.06 \mu \mathrm{m}$, Appl. Opt. 12, 700-709 (1983).

40. J. Neev, L. B. Da Silva, M. D. Feit, M. D. Perry, A. M. Rubenchik, and B. C. Stuart, "Ultrashort pulse lasers for hard tissue ablation," IEEE J. Quantum Electron. 2, 790-800 (1996).

41. G. J. R. Spooner, T. Juhasz, I. R. Traub, G. Djotyan, C. Horvath, Z. Sacks, G. Marre, D. Miller, A. R. Williams, and R. Kurtz, "Commercial and biomedical applications of ultrafast lasers," Proc. Soc. Photo. Opt. Instrum. Eng. 3934, 62-72 (2000).

42. T. Szorenyi, E. Fogarassy, C. Fuchs, J. Hommet, and F. Le Normand, "Chemical analysis of a-CNx thin films synthesized by nanosecond and femtosecond pulsed laser deposition," Appl. Phys. A 69, 941-944 (1999).

43. M. H. Niemz, "Cavity preparation with the Nd:YLF picosecond laser," J. Dent. Res. 74, 194-199 (1995).

44. A. V. Rode, E. G. Gamaly, B. Luther-Davies, B. T. Taylor, J. Dawes, A. Chan, R. M. Lowe, and P. Hannaford, "Subpicosecond laser ablation of dental enamel," J. Appl. Phys. 92, 2153-2158 (2002).

45. D. L. Matthews, L. Da Silva, B. M. Kim, and J. Marion, "Surgical applications of ultrashort pulse laser technology," in Proc. Advanced Solid State Laser Annual Meeting (Boston, MA, USA, 1999), TuA1.

46. R. Srinivasan, P. E. Dyer, and B. Braren, "Far-ultraviolet laser ablation of the cornea: photoacoustic studies," Lasers Surg. Med. 6, 514-519 (1987).

47. C. A. Pxuliato, D. Stern, R. R. Kreuger, and E. R. 
Mandel, "High-speed photography of excimer laser ablation of the cornea," Arch. Ophthal. 105, 1255-1259 (1987).

48. R. R. Anderson and J. A. Parrish, "Selective photothermolysis: precise microsurgery by selective absorption of pulsed radiation," Science 220, 524-527 (1983).

49. S. Dahan, J. M. Lagarde, V. Turlier, L. Courrech, and S. Mordon, "Treatment of neck lines and forehead rhytids with a nonablative 1540-nm Er:glass laser: a controlled clinical study combined with the measurement of the thickness and the mechanical properties of the skin," Dermatol. Surg. 30, 872-879 (2004).

50. D. Mains, G. S. Herron, R. K. Sink, H. Tanner, and R. R. Anderson, "Fractional photothermolysis: a new concept for cutaneous remodeling using microscopic patterns of thermal injury," Lasers Surg. Med. 34, 426-438 (2004).

51. V. P. Bedi, K. F. Chan, R. K. Sink, B. M. Hantash, G. S. Herron, Z. Rahman, S. K. Struck, and C. B. Zachary, "The effects of pulse energy variations on the dimensions of microscopic thermal treatment zones in nonablative fractional resurfacing," Lasers Surg. Med. 39, 145-155 (2007).

52. B. M. Hantash, V. P. Bedi, K. F. Chan, and C. B. Zachary, "Ex vivo histological characterization of a novel ablative fractional resurfacing device," Lasers Surg. Med. 39, 87-95 (2007).

53. B. M. Hantash, V. P. Bedi, V. Sudireddy, S. K. Struck, G. S. Herron, and K. F. Chan, "Laser-induced transepidermal elimination of dermal content by fractional photothermolysis," J. Biomed. Opt. 11, 041115 (2006).

54. B. M. Hantash, V. P. Bedi, B. Kapadia, Z. Rahman, K. Jiang, H. Tanner, K. F. Chan, and C. B. Zachary, "In vivo histological evaluation of a novel ablative fractional resurfacing device," Lasers Surg. Med. 39, 96-107 (2007).

55. J. N. Kabalin, "Laser prostatectomy performed with a right angle firing neodymium:YAG laser fiber at 40 watts power setting," J. Urol. 150, 95-99 (1993).

56. N. J. Barber and G. H. Muir, "High-power KTP laser prostatectomy: the new challenge to transurethral resection of the prostate," Curr. Opin. Urol. 14, 21-25 (2004).
57. R. Tooher, P. Sutherland, A. Costello, P. Gilling, G. Rees, and G. Maddern, "A systematic review of holmium laser prostatectomy for benign prostatic hyperplasia,” J. Urol. 171, 1773-1781 (2004).

58. S. P. Dretler, "Laser lithotripsy: a review of 20 years of research and clinical applications," Lasers Surg. Med. 8, 341-356 (1988).

59. K. F. Chan, G. J. Vassar, T. J. Pfefer, J. M. Teichman, R. D. Glickman, S. T. Weintraub, and A. J. Welch, "Holmium:YAG laser lithotripsy: a dominant photothermal ablative mechanism with chemical decomposition of urinary calculi," Lasers Surg. Med. 25, 22-37 (1999).

60. N. M. Fried, "Thulium fiber laser lithotripsy: an in vitro analysis of stone fragmentation using a modulated 110-watt thulium fiber laser at 1.94 microm," Lasers Surg. Med. 37, 53-58 (2005).

61. H. Lee, H. W. Kang, J. M. Teichman, J. Oh, and A. J. Welch, "Urinary calculus fragmentation during Ho: YAG and Er:YAG lithotripsy," Lasers Surg. Med. 38, 39-51 (2006).

62. H. Lee, J.-W. Yoon, Y.-D. Jung, J.-H. Kim, T. R. Robert, M. H. J. Teichman, and A. J. Welch, "Comparison of sapphire and germanium fibers for erbium: YAG lithotripsy,” J. Opt. Soc. Korea 12, 309-313 (2008).

63. C. A. Chaney, Y. Yang, and M. Fried, "Hybrid germanium/ silica optical fibers for endoscopic delivery of erbium: YAG laser radiation," Lasers Surg. Med. 34, 5-11 (2004).

64. Y. Yang, C. A. Chaney, and N. M. Fried, "Erbium: YAG laser lithotripsy using hybrid germanium/silica optical fibers,” J. Endourol. 18, 830-835 (2004).

65. T. J. Polletto, A. K. Ngo, A. Tchapyjnikov, K. Levin, D. Tran, and N. M. Fried, "Comparison of germanium oxide fibers with silica and sapphire fiber tips for transmission of erbium:YAG laser radiation," Lasers Surg. Med. 38, 787-791 (2006).

66. K. Iwai, Y. W. Shi, K. Nito, Y. Matsuura, T. Kasai, M. Miyagi, S. Saito, Y. Arai, N. Ioritani, Y. Okagami, M. Nemec, J. Sulc, H. Jelinkova, M. Zavoral, O. Kohler, and P. Drlik, "Erbium:YAG laser lithotripsy by use of a flexible hollow waveguide with an end-scaling cap," Appl. Opt. 42, 2431-2435 (2003). 